\title{
Average Subtraction Method for Image Reconstruction of Brain using ECVT for Tumor Detection
}

\author{
Rizky Maharani1 ${ }^{1}$ Rizki Edmi Edison ${ }^{1 *}$, Muhammad Fathul Ihsan ${ }^{2}$, Warsito Purwo Taruno ${ }^{2}$ \\ ${ }^{1}$ Neuroscience Center - University of Muhammadiyah Prof Dr. HAMKA, Gandaria IV no. 24, South Jakarta \\ 12120, Indonesia \\ ${ }^{2}$ CTECH Labs Edwar Technology, Jalur Sutera Kav. Spektra Blok 23 BC No. 10-12, Alam Sutera, Tangerang, \\ Banten 15320, Indonesia
}

\begin{abstract}
Brain Electrical Capacitance Volume Tomography (Brain ECVT) is a new technique that realizes real-time volumetric imaging of dynamic changes in electrical activity of the brain and allows total interrogation of the whole volume inside a helmet-shaped sensor. The technique has been used for investigating numerous brain functional abnormalities, including brain tumors, based on permittivity different from the tumor case, as compared to the normal brain. However, interpretation of the conventional Brain ECVT image is not practical for clinical purposes, as the image resolutions are high in the cortical area and lower in the middle region. The technique provides relatively good sensitivity when the tumor is located near the cortex. In this study, we developed a novel method, namely the average subtraction technique, to process the reconstructed image of the brain obtained by Brain ECVT. The technique generates a three-dimensional intracranial distribution of permittivity that correlates with the electrical activity map of the brain with improved resolution in the center region of the brain. The technique provides better insight into brain intracranial electrical activity, which is quite distinctive when there is tumor development inside the brain. The technique may lead to better detection of brain tumors inside the brain based on the electrical activity scan.
\end{abstract}

Keywords: Brain ECVT; Brain tumor; Image reconstruction

\section{Introduction}

In neurosciences, non-invasive imaging methods could provide useful information in two broad areas: imaging of variations or abnormalities in structure and imaging of normal or abnormal functional activity of the brain. While the coding of information in brain pathways has long been of interest in this field, the lack of methods capable of measuring the brain structure in a simple and non-invasive fashion has hampered research. The ease of diagnosing structural abnormalities in neurology has been transformed since the development of X-ray CT in the 1970s and, more recently, MRI.

Real-time volumetric imaging of human brain structural abnormalities based on the electric field was proposed for the first time in 2013, with a device called a fourdimensional brain electrical capacitance volume tomography (4D Brain ECVT; Taruno et al., 2013a). ECVT is an advanced electrical capacitance tomography (ECT), a technique for

${ }^{*}$ Corresponding author's email: rizkiedmiedison@uhamka.ac.id,, Tel.: +6285664666124 doi: 10.14716/ijtech.v11i5.4325 
obtaining information about the distribution of the contents of closed pipes or vessels by measuring variations in the dielectric properties of the material inside the vessel. Typical information of the cross-sectional or two-dimensional images obtained in ECT has been upgraded to volumetric imaging that can be measure by the ECVT system with a non-linear change in electric field distribution (Warsito et al., 2007; Wang et al., 2010). Volume tomography is the only technique that is able to realize real-time volumetric imaging of dynamic changes in dielectric materials and allows total interrogation of the whole volume within the sensor domain with an arbitrary shape of the geometry, i.e. a helmet shape sensor array for head scans.

However, brain tumors are already known to have dielectric properties and different permittivity from the normal brain (Yoo, 2004). Generally, an abnormal mass in the brain may cause propagation signals that reach the cortex of the brain. Furthermore, the phenomenon named "brain signal death" will decrease the signals detected by the ECVT sensor (Taruno et al., 2013b) producing specific image construction. However, interpreting such images is not yet practical. In this research, we propose an alternative algorithm for image reconstruction that will make it easier to interpret brain tumor detection by using Brain ECVT.

\section{Methods}

\subsection{Subject}

The study was conducted on five brain tumor patients who came to Edwar Health Care. A written consent form was acquired from each patient. The inclusion criteria included having no medical issue other than the intracranial tumor, having never been operated on before, the tumor being located in the supratentorial region, and the presence of a single tumor. The reconstructed image on the brain tumor by Brain ECVT was then compared to the gold standard images, such as X-ray CT scans.

\subsection{Brain ECVT System Configuration}

The Brain ECVT system consists of three main components: the data acquisition system (DAS) for measuring capacitance, the helmet sensor to receive capacitance signals (Figure 1a), and the PC for data acquisition control and image reconstruction. The capacitance measurements are conducted between pairs of electrodes placed on the outside wall of the sheet covering the head and installed within the helmet shape sensor. The capacitance data is a response to the change of permittivity electrodes placed on the helmet, and there are 496 independent capacitance values (Figure 1b).

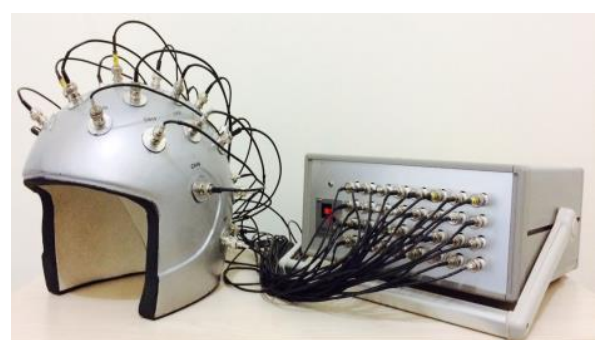

(a)

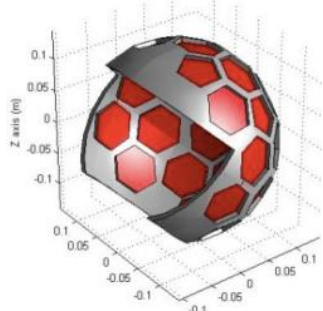

(b)

Figure 1 (a) Helmet sensor of Brain ECVT and DAS; (b) Sensor configuration

The difference of potential measured in the ECVT determines the measured capacitance value, which is also related to the permittivity distribution of the measured 
dielectric material. From the simple capacitance and the Poisson equation, the capacitance value can be written mathematically as follows:

$$
C_{i}=-\frac{1}{\Delta V_{i}} \oiint_{A_{i}} \varepsilon(x, y, z) \nabla V(x, y, z) d A
$$

where $C_{i}$ is measured capacitance value on the $i^{\text {th }}$ electrode pair, $A_{i}$ is the detector area, and $\varepsilon$ and $V$ are permittivity and electrical potential distribution, respectively. However, the measured capacitance variable with the permittivity distribution variable to this volume shows the case of nonlinear calculations. One of the linear approximation methods for Equation 1 is to use the sensitivity value approach as follows:

$$
S_{i j} \cong \operatorname{Voxel}_{j} \frac{E_{s i}(x, y, z) \cdot E_{d i}(x, y, z)}{V_{s i} V_{d i}}
$$

where $E_{s i}$ is the distribution of the electric field when both electrode-pair plates are given a voltage $V_{s i}$. Whereas $E_{d i}$ is the distribution of the electric field when both plates are given a voltage $V_{d i}$. Voxel $_{j}$ is the $j^{\text {th }}$ voxel element. The sensitivity of the matrix itself is a picture of the electric field intensity from a measurement chamber that has a certain capacitance region. The aim is to determine the location of the distribution of capacitance positions against the sensor measurement area. With the sensitivity value from Equation 2 and the measured capacitance value from Equation 1, a linear approach to reconstruct the capacitance can be obtained:

$$
C=S \cdot G
$$

where $C, S$, and $G$ are capacitance, sensitivity matrix obtained by simulation using Equation 2 , and permittivity, respectively. There are two approaches for solving equation: forward problem and inverse problem. The forward problem approach finds the capacitance matrix when given the permittivity matrix and sensitivity matrix, while the inverse problem approach finds permittivity distribution when given the measured capacitance value and sensitivity matrix (Taruno et al., 2014). Equation 3 is given as follows:

$$
G=S^{T} C
$$

where $G$ is the permittivity distribution that will be reconstructed. The sensitivity matrix $S$ is being transposed $S^{T}$.

\subsection{Experiment}

The procedure for using Brain ECVT consists of four stages, namely warming up the system (set port number, channel number, and matrix sensitivity), calibration, data acquisition, and post-processing. Data *.dat used in this study is data from one type of experiment, meaning that at the warming-up stage, the port number settings, the number of channels, and the matrix sensitivity used are the same. The difference lies in the calibration and post-processing stages. The patients were recorded by a 32-channel data acquisition system (DAS) DAQ01201205V with data acquisition speeds of \pm 5.6 seconds per frame, and it was manufactured by CTECH Labs Edwar Technology, Indonesia. The patients did not perform any tasks while sitting in a chair for \pm two minutes. The helmet sensor was placed gently on the head and comfortably secured (Figure 2). 

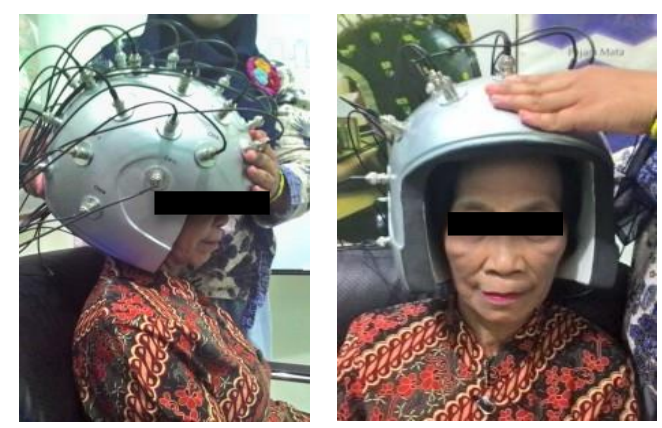

Figure 2 Placement of helmet sensor on the head of the patient during recording

\subsection{Image Reconstruction}

Originally, the alternative ECVT image reconstruction method was adapted from the previous study (Taruno et al., 2014), where one of the steps of image reconstruction in that study was substituted at the conventional ECVT image reconstruction step. However, there is a difference between the intended use of this step. The previous study uses the reconstruction step to display ECVT images that show the location of the motor activities of the human brain when given a task in the motor brain function area, whereas in this study the reconstruction step aims to display abnormalities (in this case intracranial tumor mass). After substituting these steps, an alternative method is created, called the Average Subtraction Method.

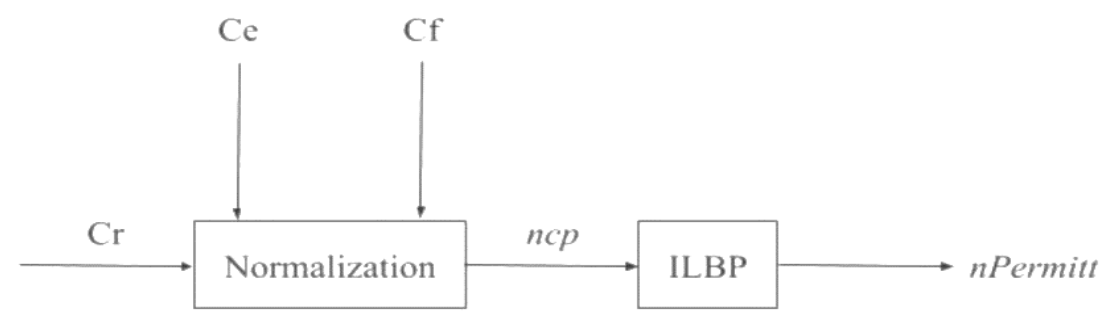

Figure 3 Diagram of ECVT conventional image reconstruction method

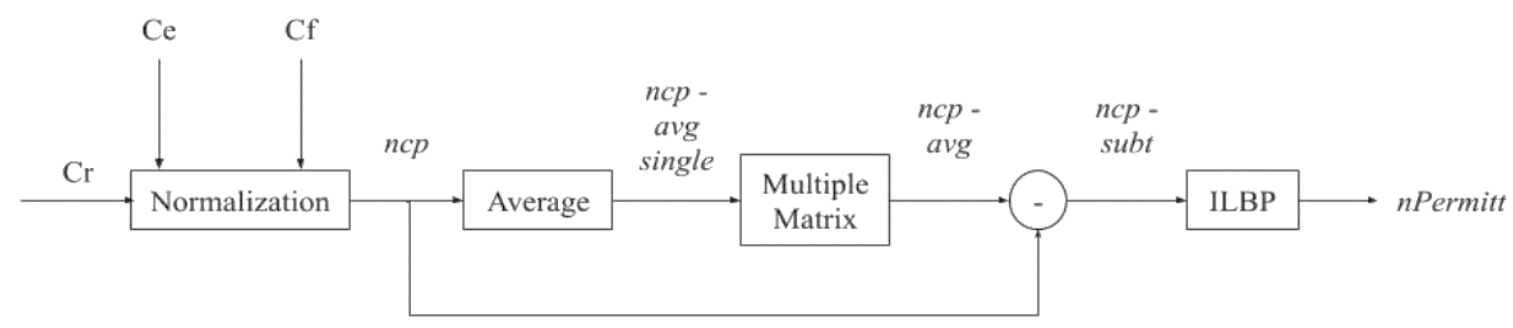

Figure 4 Diagram of ECVT average subtraction image reconstruction method

The difference between the conventional and average subtraction image reconstruction methods can be seen by comparing the diagrams from Figures 3 and 4 . The coefficients of Ce and Cf, the capacitance data of air and water objects, respectively have a matrix multiplication $[1 \times \mathrm{m}]$. While the $\mathrm{Cr}$ coefficient is the capacitance data of human head objects with matrix multiplication $[\mathrm{f} \times \mathrm{m}]$, the notion of $\mathrm{f}$ itself is the number of frames taken. The coefficient of $\mathrm{m}$ is the number of measurements according to the following formula, where $\mathrm{Ne}$ is the number of electrodes. Since the experiment used a 32-electrode sensor, the number of measurements was 496. 


$$
\mathrm{m}=\frac{\mathrm{N}_{\mathrm{e}}\left(\mathrm{N}_{\mathrm{e}}-1\right)}{2}
$$

Because the capacitance values obtained from each sensor pair are extremely diverse, pairs that are close to each other will have a much higher capacitance value than the pairs that are far apart; therefore, a normalization of the measured capacitance value is carried out. Normalization uses the capacitance value when the sensor contains material with low permittivity, namely air, and the capacitance value when the sensor contains material with high permittivity, namely water (data from the calibration stage). The normalized capacitance value $C_{i j}$ is calculated by the following equation:

$$
C_{i j}=\sum_{i}^{N e} \sum_{j=i+1}^{N e} \frac{C_{i, j}^{m e a s}-C_{i, j}^{e}}{C_{i, j}^{f}-C_{i, j}^{e}}
$$

where $C_{i, j}^{\text {meas }}$ is the capacitance measured between electrodes $\mathrm{i}$ and $\mathrm{j}$ with the head object, $C_{i, j}^{e}$ is the capacitance between electrodes $i$ and $j$ with the air object, and the area $C_{i, j}^{f}$ is the capacitance between electrode pairs $i$ and $j$ with the water object.

After the normalized capacitance value (ncp) is obtained in the form of matrix multiplication [ $\mathrm{f} \times \mathrm{m}$ ], then the value is limited to a value between 0 and 1.5 , assuming the capacitance value obtained from the water object must be greater than the value of the capacitance obtained from the air object, and there is the possibility of error measuring capacitance, where the head object's capacitance is slightly smaller than the air object's capacitance; however, this error can be ignored. After obtaining the ncp value, the ILBP algorithm reconstruction stage is used to convert the ncp to normalized permittivity. The result of the ILBP algorithm reconstruction is a normalized permittivity matrix (nPermitt) with matrix multiplication [ $\mathrm{f} \times \mathrm{n}$ ], where $\mathrm{n}$ is the total voxel of $32768(32 \times 32 \times 32$ pixels $)$.

In the conventional method, the reconstruction technique is completed with these two steps; however, the difference is that the average subtraction method has three other stages after normalization and before using the ILBP algorithm reconstruction stage. In the average subtraction method, after the ncp value is obtained, then the average (average process) is searched for each frame. Because it is averaged per frame, the result obtained is a vector of $[1 \times \mathrm{m}]$, which is called the ncp-avg single. Before it can be disputed with the original ncp value, a Multiple Matrix (Mult Mat) is first performed to change the vector shape $[1 \times \mathrm{m}]$ to the matrix form [ $\mathrm{f} \times \mathrm{m}]$ so that it can be disputed, and the result of the multiplication is called ncp-avg. After reducing the value of ncp with ncp-avg (subtraction process) the ncp-subt value is obtained by matrix multiplication [ $\mathrm{f} \times \mathrm{m}]$, and then the ncpsubt value is applied to the ILBP algorithm reconstruction stage. The results are also the same as those of conventional reconstruction methods, which are in the form of normalized permittivity values ( $n$ Permitt) with matrix multiplication [ $\mathrm{f} \times \mathrm{n}]$.

\section{Results and Discussion}

Brain tumors that arise from different cells both from within the brain and systemic tumors that have metastasized to the brain are among the top 10 causes of cancer-related deaths (Wrench et al., 2002). Patients with brain tumors may present for care with a variety of signs and symptoms. The clinical manifestations of brain tumors are usually referable to the anatomic area of the brain and involved or adjacent structures (Walker et al., 2013). Headache is a common clinical feature in patients in the emergency room and in general neurology clinics. Usually, headache is accompanied by further neurological deficits, such as seizure, fatigue, and cognitive dysfunction (Hamilton et al., 2007). Non-experienced 
physicians in headache disorders are regularly confronted with the question of whether it is necessary to perform neuroimaging to confirm a distinct headache diagnosis and diagnose underlying brain pathology (Holle and Obermann, 2013). Currently, with appropriate and accepted clinical evidence, MRI efficiently makes the initial diagnosis of brain tumors (Butowski, 2015). However, this kind of neuroimaging technique is difficult to justify in a resource-restricted medical environment. Diagnosis is generally made after a long duration of symptoms, although the brain tumor is a common neurosurgical condition, which may have a negative impact on treatment outcome (Bunyaratavej et al., 2010).

To detect brain tumors early after the onset of symptoms, a non-invasive, real-time neuroimaging system must be developed that is easy to use, safe, and inexpensive. The ideal method would be a device that could produce three-dimensional images of brain tumors. Geared toward such a specific improvement in the neuroimaging area, Brain ECVT was developed for specific features (Berawi, 2015). The advantages of Brain ECVT are that it is relatively inexpensive, safe, non-invasive, portable, and real-time. The main drawback is a relatively poor spatial resolution. While its spatial resolution will probably improve as technical advances are made, the technique must always be limited by the fact that the electric field spreads throughout the whole subject so that the inverse problem is less well defined than in X-ray CT or MRI. However, its advantages may still enable it to be indispensable for monitoring structural changes at the bedside, in casualty departments, or in remote locations where large scanners are too expensive or impractical. If neuroimaging with Brain ECVT is successful, then it could be used in several key clinical areas in which other methods of functional brain imaging are unsuited. Brain ECVT can further generally assist technicians in advancing their qualifications in the health and medical expertise field (Elfani and Putra, 2013).

(a)
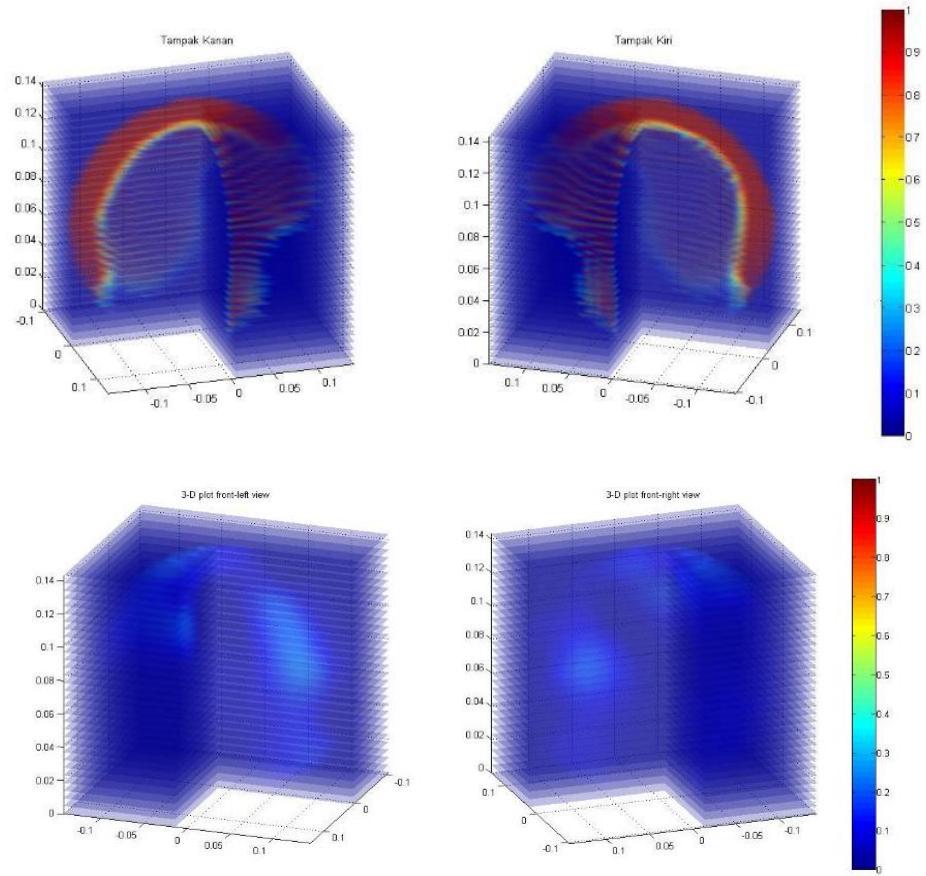

Figure 5 Brain ECVT 3D-image results from conventional reconstruction method (a) and average subtraction (b) from a subject without intracranial abnormalities 


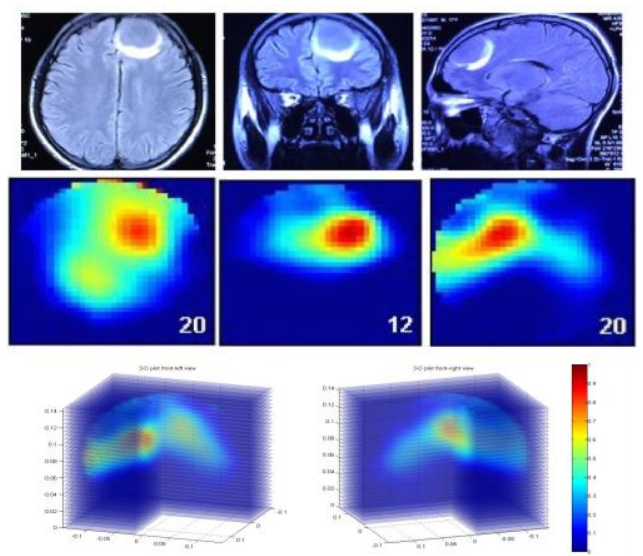

Figure 6 Image comparison of brain tumor detected by X-ray CT or MRI with Brain ECVT Average Subtraction method image reconstruction
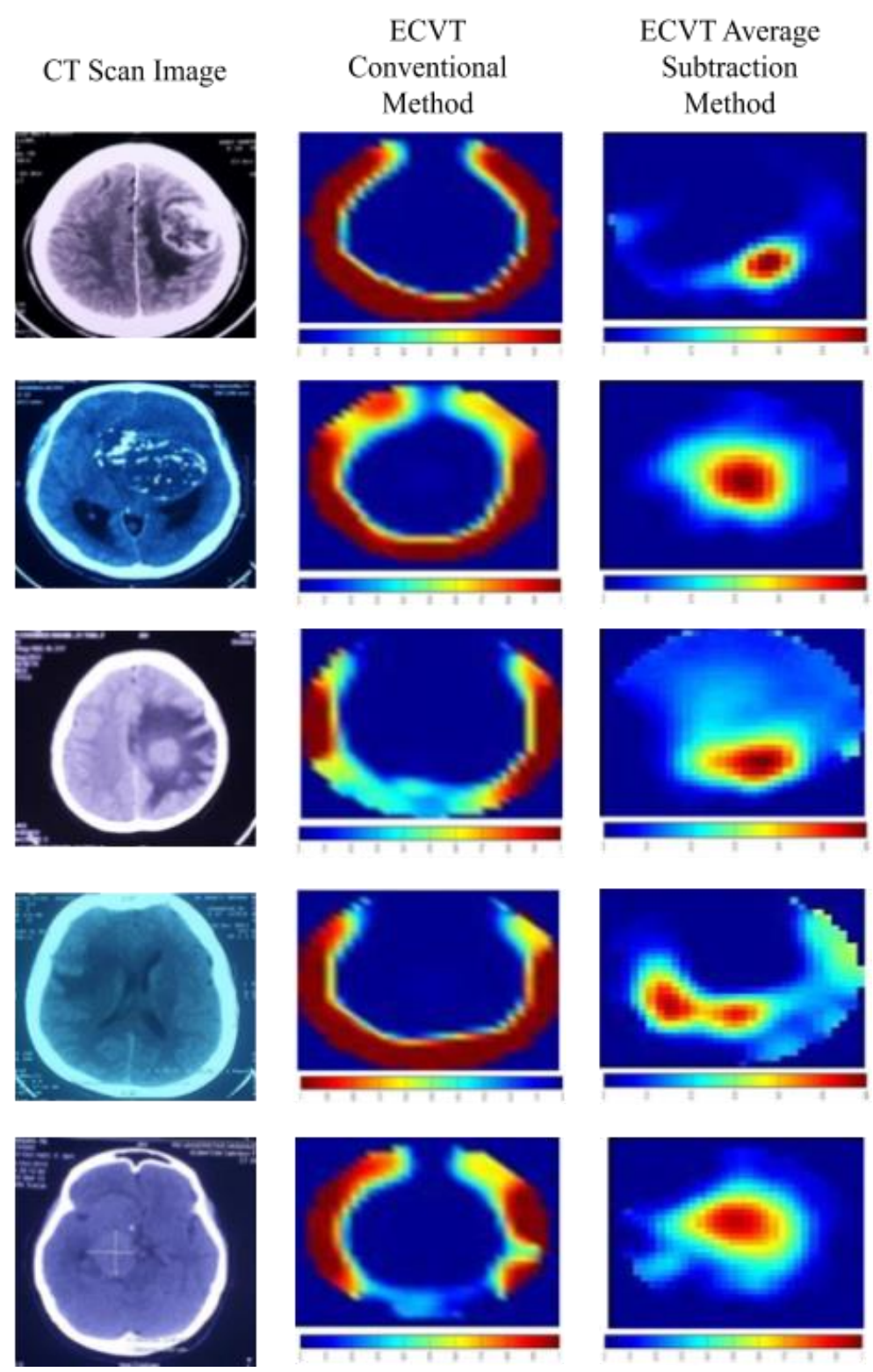

Figure 7 Axial image comparison of brain tumor detected X-Ray CT, Brain ECVT with conventional image construction, and Brain ECVT with average subtraction image reconstruction 
Figures 6 and 7 compare the CT scan image, the image result of the conventional reconstruction method, and the image result of the average subtraction reconstruction method. All of them are in the form of axial cuts (in the direction of the z-axis, 2D). An obvious difference from the results of the conventional reconstruction method images and the average subtraction reconstruction is that the images do not form the surface of the sensor on the image from the average subtraction reconstruction method. This is because, in the conventional method, there is a stage before the reconstruction of the algorithm where the ncp value less than 0 will be considered 0 , and a value greater than 1 will be considered 1; whereas in the average subtraction method, before being made 0 , the ncp values undergo a subtraction process first, causing the possibility of ncp values smaller than 0 that change to greater than 0 . The possibility of values greater than 0 is what causes the appearance of red in the middle of the sensor space (conventional red image on the sensor surface).

In addition, this elimination process also causes the possibility of values greater than 0 that have diminished in value because they are reduced by the average value so that the red color that appears on the sensor surface does not appear in the average subtraction image, and the blue color inside the sensor surfaces, such as conventional images, is also not entirely blue (visible from yellow to red) in the average subtraction image. By comparison, the ncp value equal to 0 appears more in conventional (visible from blue more than red in the image), while the ncp value that is not equal to 0 appears more in average subtraction (visible from yellow to red, while the color blue is not as blue in the conventional image). In the average subtraction image, the dark yellow to red colors that appear around the middle of the sensor show the correlation of the tumor location. The permittivity scale above 0.5 to 0.9 that appears on the image of objects with intracranial tumors shows the location of intracranial tumors following information from CT scans of the tumor patients. Determination of the permittivity scale (color scale) and the lateralization of the location of areas that have a high permittivity distribution are the main modalities of the average subtraction reconstruction method to help image interpretation.

According to image analysis, the color scale represents the permittivity distribution that has been reconstructed. The reconstructed permittivity distribution is the permittivity distribution that is processed by the ECVT to image brain anatomy and brain activity. When formulated, it will produce the following equation:

$$
\varepsilon(r, t)=\varepsilon_{0}(r)+\varepsilon^{\prime}(r, t)
$$

where $\varepsilon(r, t)$ is the reconstructed permittivity distribution, which is influenced by the position $(r)$ and time $(t)$ functions. Whereas $\varepsilon_{0}(r)$ is the permittivity distribution by the brain cell itself (symbolizing brain anatomy), where it is not influenced by the function of time because the brain is considered to be in a constant state and because $\varepsilon^{\prime}(r, t)$ itself is the permittivity distribution of brain activity. The variable $\varepsilon(r, t)$ is also the permittivity distribution reconstructed by ECVT; thus, the form of Equation 7 is the various variables processed by ECVT to produce ECVT images.

In previous studies, the average subtraction reconstruction method was applied to see the motor activity of the brain when given a task (Taruno et al., 2014). The aim is to determine whether the ECVT is also able to show brain activity in the area of motor function when an object is given and not given a task. Meanwhile, this study shows the location of the abnormal object (tumor mass) in the brain. If we complete the mathematical equation of the average subtraction reconstruction method, then the equation is as follows: 


$$
\begin{array}{r}
\overline{\varepsilon^{\prime}(r, t)}=\overline{\varepsilon(r, t)}-\overline{\varepsilon_{0}(r)} \\
\varepsilon^{\prime \prime}(r, t)=\varepsilon^{\prime}(r, t)-\overline{\varepsilon^{\prime}(r, t)}
\end{array}
$$

Equation 8 is the average of the same variables as Equation 7 , which is then entered into Equation 9 to produce $\varepsilon^{\prime \prime}(r, t)$ which is the permittivity distribution of the ECVT reconstructed using the average subtraction method, producing images such as Figure 5 (for normal cases). However, the result of $\varepsilon^{\prime \prime}(r, t)$ has two possibilities, particularly $\varepsilon^{\prime \prime}(r, t) \approx 0$, which is considered to be the case of a normal brain object (without tumor mass), and $\varepsilon^{\prime \prime}(r, t) \gg 0$ is considered a case of an abnormal brain object (with a tumor mass). In Figure 6, the red color that appears in the right position has a value with a permittivity scale greater than 0 , which indicates a position that corresponds to the location of the patient's intracranial tumor mass.

In referring to Taruno et al. (2013b) to determine the presence of brain tumors from the results of the conventional method, tumor can be detected by the presence of a relatively weak electrical activity zone on the cortical area in the Brain ECVT image. To show the difference between normal and abnormal subjects (i.e., those with and without a brain tumor), it has been proven that there are significant differences. The results of conventional image reconstruction method should have spots of a low normalized permittivity distribution value, which indicates weak brain activity due to a tumor that causes functional abnormalities of that particular brain region to appear. However, this does not always appear in the results of image reconstruction with the assumption due to several problem factors in the image reconstruction method. In Figure 7, two out of five conventional image results (the first and fourth cases) did not show any difference from the image subject without a brain tumor, and there should be several spots like the conventional image results in the second, third, and fifth cases. Furthermore, this method has not been able to indicate where tumor mass is located, consequently making it even harder to interpret the image. In contrast to the results of the image average subtraction reconstruction method, the image interpretation process becomes more apparent because it not only directly determines the presence or absence of a brain tumor mass but also tells its position. In five out of five cases in Figure 7, the tumor position can already be classified based on the right or left location. However, further work to locate the brain tumor position more precisely using the average subtraction method is possible. The purpose of forming this industry (CTECH Labs EdWar Technology) and university (University of Muhammadiyah professor Dr. HAMKA) collaboration is to ensure success (Hanid et al., 2019).

\section{Conclusions}

In this study, we developed an image reconstruction method, called average subtraction, and applied it to a brain image obtained by Brain ECVT. The technique provides a three-dimensional distribution of permittivity that correlates with the electrical activity of the whole brain with an improved resolution, particularly in the center region of the brain. The technique showed a distinctive difference in the electrical activity of the intracranial brain with tumor cases, as compared to the normal brain. The technique may lead to better detection of brain tumors based on an electrical activity scan using ECVT. Further quantification of electrical activity differences is still needed for tumor detection, as the electrical activity may also vary in the presence of brain stimulations or other abnormalities that could affect brain electricity. 


\section{References}

Berawi, M.A., 2015. Technology Breakthrough: A Need for Continuous Improvement. International Journal of Technology, Volume 6(3), pp. 302-305

Bunyaratavej, K., Siwanuwatn, R., Chantra, K., Khaoroptham, S., 2010. Duration of Symptoms in Brain Tumors: Influencing Factors and Its Value in Predicting Malignant Tumors. Journal of the Medical Association of Thailand, Volume 93(8), pp. 903-910

Butowski, N.A., 2015. Epidemiology and Diagnosis of Brain Tumors. Continuum, Volume 21(2 Neuro-oncology), pp. 301-313

Elfani, M., Putra, N.K., 2013. Biomedical Engineering and Its Potential for Employment in Indonesia. International Journal of Technology, Volume 4(1), pp. 34-44

Hamilton, W., Kernick, D., 2007. Clinical Features of Primary Brain Tumors: A Case-Control Study using Electronic Primary Care Records. The British Journal of General Practice, Volume 57(542), pp. 695-699

Hanid, M., Mohamed, O., Othman, M., Danuri, M.S.M., Mei Ye, K., Berawi, M.A., 2019. Critical Success Factors (CSFS) in University-Industry Collaboration (UIC) Projects in Research Universities. International Journal of Technology. Volume 10(4), pp. 667-676

Holle, D., Obermann, M., 2013. The Role of Neuroimaging in the Diagnosis of Headache Disorders. Therapeutic Advances in Neurological Disorders, Volume 6(6), pp. 369-374

Taruno, W.P., Baidillah, M.R., Sulaiman, R.I., Ihsan, M.F., Fatmi, S.E., Muhtadi, A.H., Haryanto, F., 2013a. 4D Brain Activity Scanner using Electrical Capacitance Volume Tomography (ECVT). In: Biomedical Imaging (ISBI), IEEE $10^{\text {th }}$ International Symposium on, San Francisco, pp. 1006-1009

Taruno, W.P., Baidiliah, M.R., Sulaiman, R.I., Ishan, M.F., Yusaf, A., Widada, W., Aljohani, M., 2013b. Brain Tumor Detection using Electrical Capacitance Volume Tomography (ECVT). In: Neural Engineering, 6 ${ }^{\text {th }}$ International IEEE EMBS Conference, pp. 743-746

Taruno, W.P., Ihsan, M.F., Baidiliah, M.R., Tandian, T.A., Mahandra, M., Aljohani, M., 2014. Electrical Capacitance Volume Tomography for Human Brain Motion Activity Observation. In: Middle East Conference on Biomedical Engineering (MECBME), pp. 147-150

Walker, D., Hamilton, W., Waller, F.M., Watts, C., 2013. Strategies to Accelerate Diagnosis of Primary Brain Tumors at the Primary-Secondary Care Interface in Children and Adults. CNS Oncology, Volume 2(5), pp. 447-462

Wang, F., Marashdeh, Q., Fan, L.S., Warsito, W., 2010. Electrical Capacitance Volume Tomography: Design and Applications. Sensors, Volume 10(3), pp. 1890-1917

Warsito, W., Marashdeh, Q., Fan, L.S., 2007. Electrical Capacitance Volume Tomography. Sensor Journal, Volume 27(4), pp. 525-535

Wrench, M., Minn, Y., Chew, T., Bondy, M., Berger, M., 2002. Epidemiology of Primary Brain Tumors: Current Concepts and Review of the Literature. Neuro-Oncology, Volume 4(4), pp. 278-299

Yoo, D.S., 2004. The Dielectric Properties of Cancerous Tissues in a Nude Mouse Xenograft Model. Bioelectromagnetics, Volume 25 (7), pp. 492-497 